Supplementary Information for:

\title{
Colloidal $\boldsymbol{n}$-doped CdSe and CdSe/ZnS Nanoplatelets
}

Lifeng Wang ${ }^{1,2}$, Dongmei Xiang ${ }^{1}$, Kaimin Gao ${ }^{1,2}$, Junhui Wang ${ }^{1,2^{*}}$, Kaifeng Wu ${ }^{1,2}$

${ }^{1}$ State Key Laboratory of Molecular Reaction Dynamics and Dynamics Research

Center for Energy and Environmental Materials, Dalian Institute of Chemical

Physics, Chinese Academy of Sciences, Dalian, Liaoning 116023, China

${ }^{2}$ University of Chinese Academy of Sciences, Beijing 100049, China

*Corresponding Author: wjh@dicp.ac.cn

\section{Contents list:}

Figures S1-S12

Sample preparations

Photochemical doping of NPLs

TA and $\mathrm{u}-\mathrm{PL}$ experimental setups

Supplementary Notes 
a
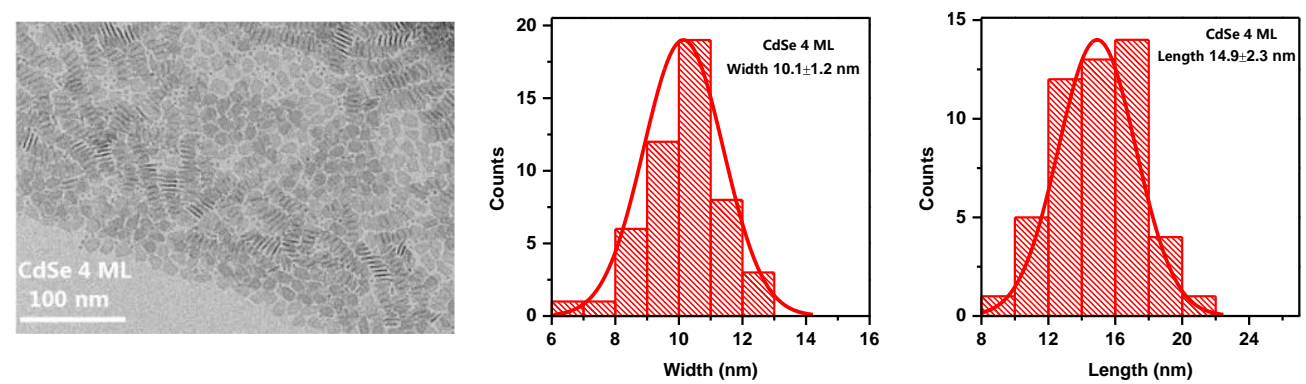

b
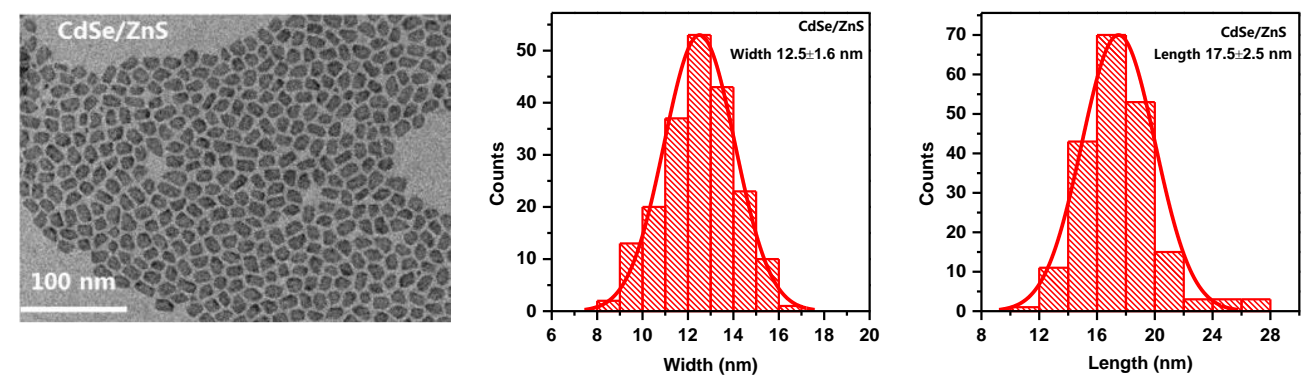

C
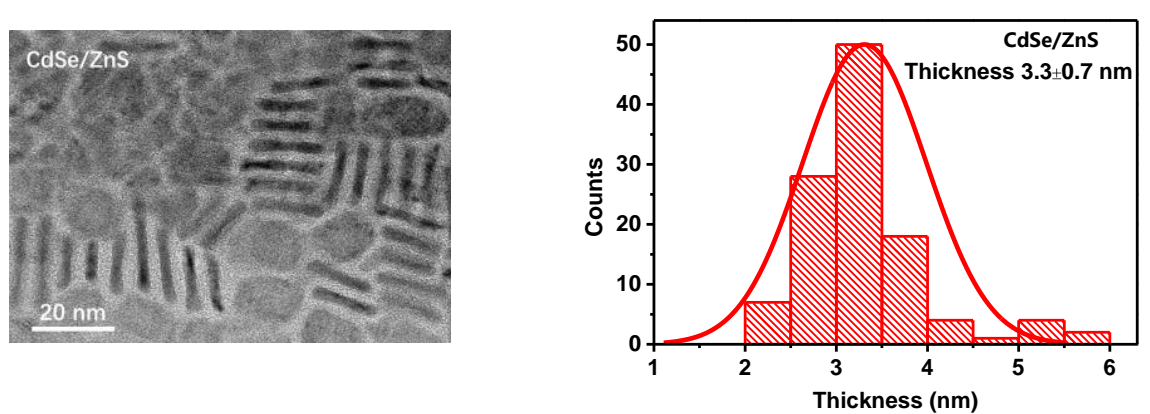

Figure S1. (a, b) TEM images of NPLs (left), histograms for the NPL's width (middle), and length (right) for (a) 4 ML CdSe NPLs and (b) CdSe/ZnS NPLs, respectively. (c) TEM images of CdSe/ZnS NPLs and histograms for its thickness. The average thickness of the $\mathrm{ZnS}$ shell is $\sim 0.9 \mathrm{~nm}$, which is obtained by subtracting the thickness of 4 ML NPLs ( $1.5 \mathrm{~nm})$ from that of CdSe/ZnS NPLs $(\sim 3.3 \mathrm{~nm})$. 
a

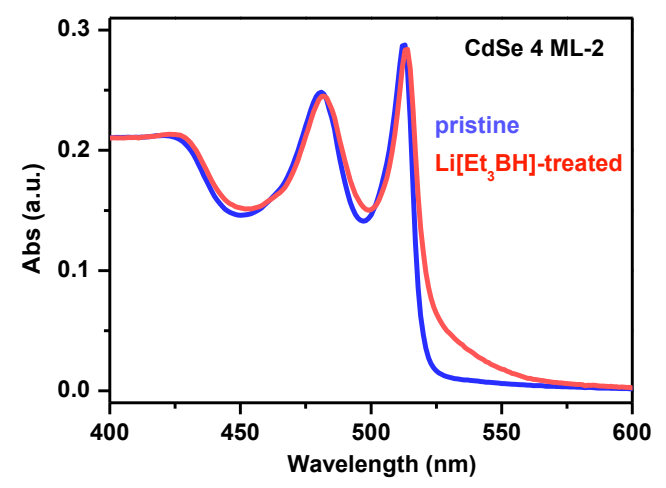

b

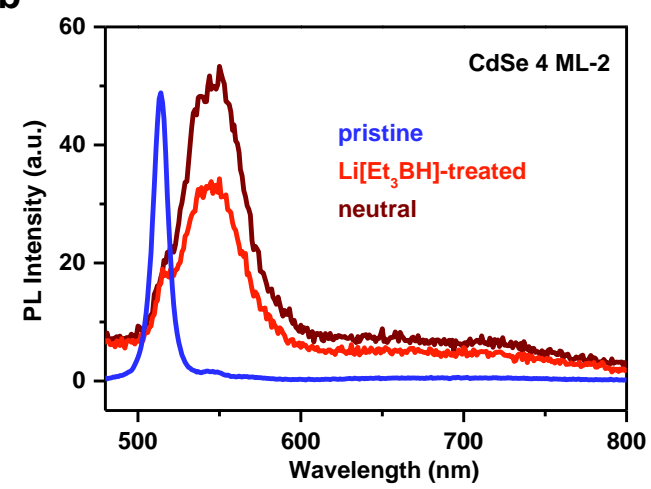

Figure S2. Absorption and PL spectra of 4 ML CdSe NPLs which was washed with ethanol to reduce the amount of surface ligands (labeled as CdSe 4 ML-2). (a) Absorption spectra of pristine (blue line) and $\mathrm{Li}\left[\mathrm{Et}_{3} \mathrm{BH}\right]$ treated (red line) CdSe 4 ML-2 NPLs. (b) PL spectra of pristine (blue line), Li[Et $3 \mathrm{BH}]$ treated (red line), and neutralized (exposed to the air) CdSe 4 ML-2 NPLs (dark red line).

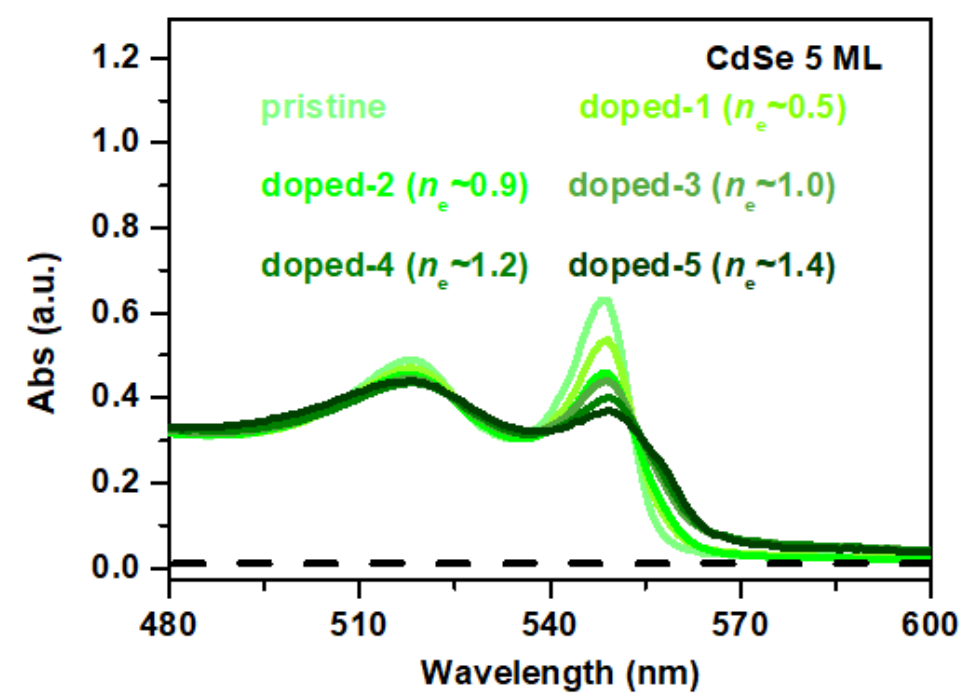

Figure S3. Absorption spectra of pristine and $n$-doped NPLs with various doping levels for 5 ML CdSe NPLs. 

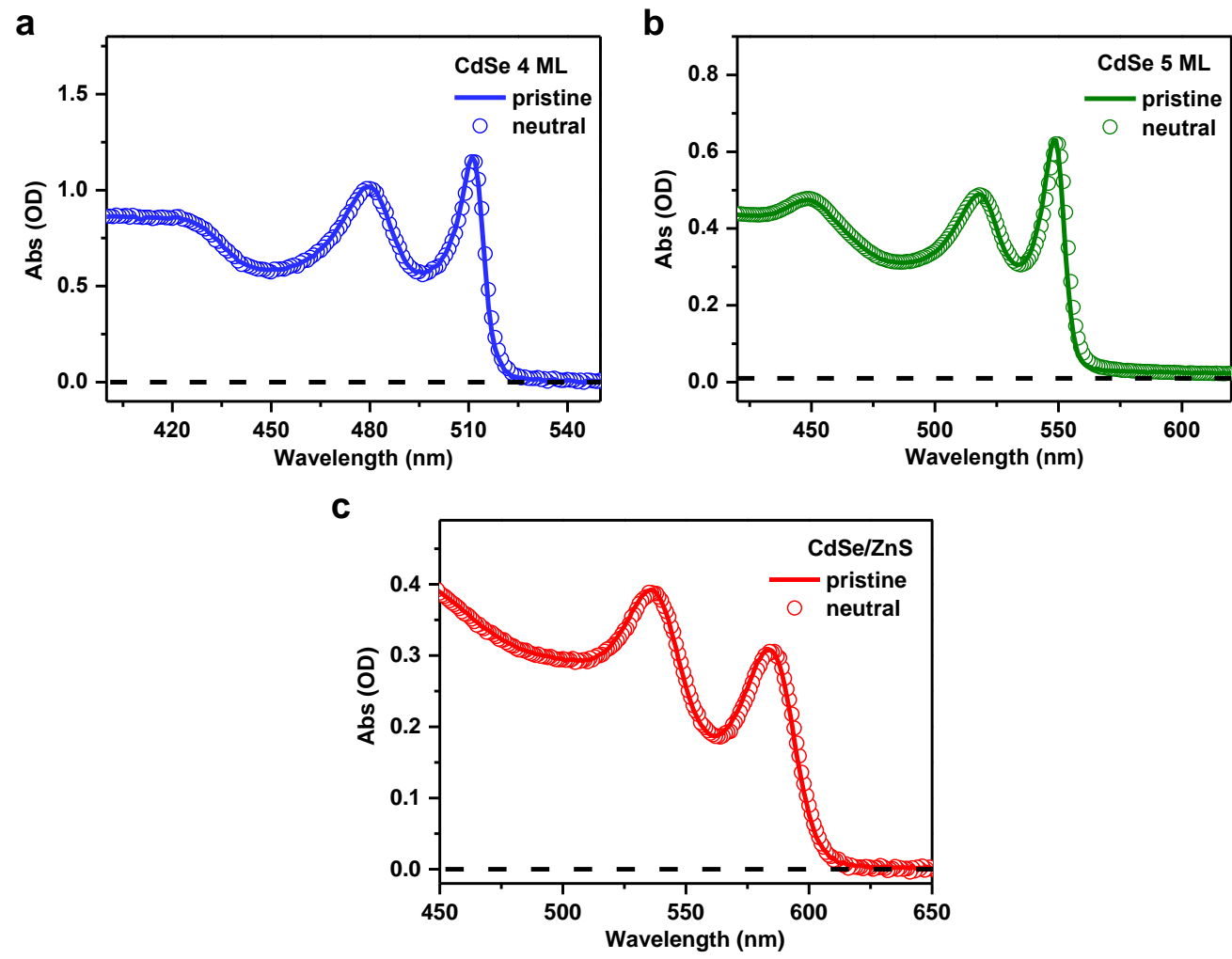

Figure S4. Absorption spectra of pristine (solid lines) and neutral (circles) NPLs for (a) 4 ML CdSe, (b) 5 ML CdSe, and (c) CdSe/ZnS NPLs, separately.

a

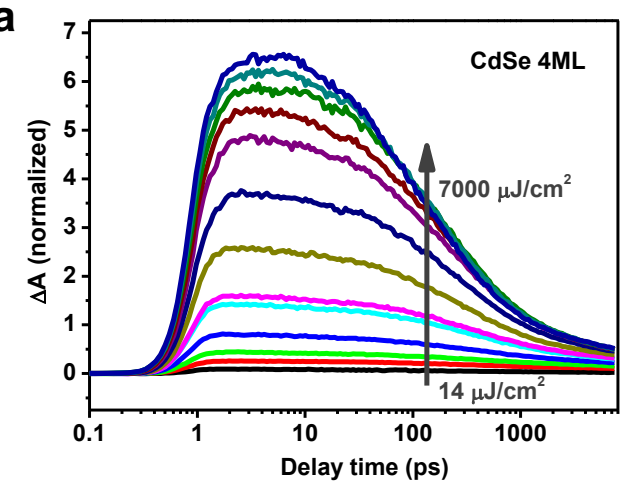

C

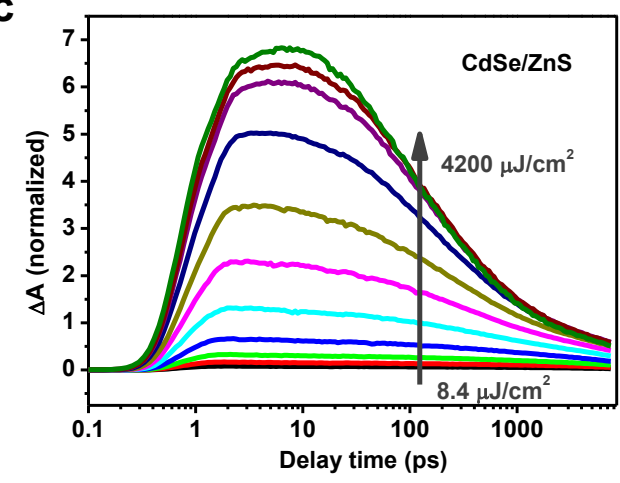

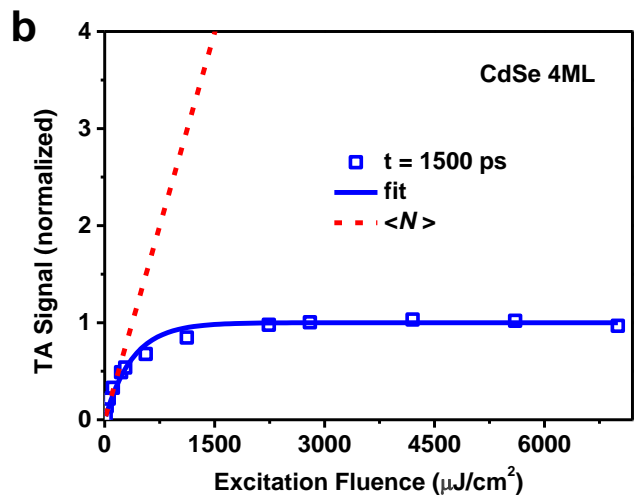

d

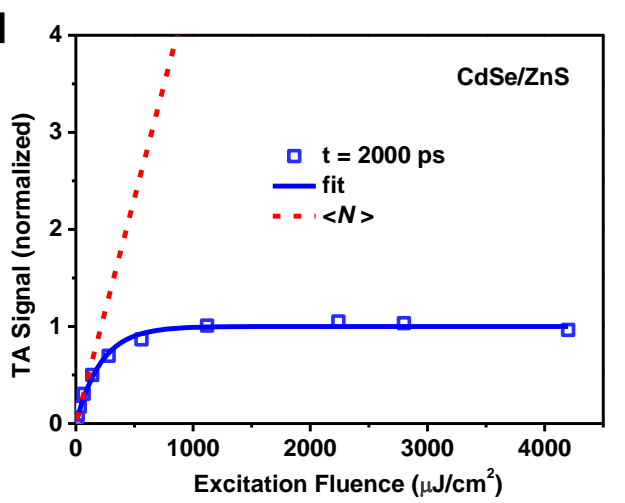


Figure S5. TA kinetics probed at the e-hh transition feature of NPLs for (a) $4 \mathrm{ML}$ CdSe and (c) CdSe/ZnS NPLs. They are excited with various pump fluences from $14 \mu \mathrm{J} / \mathrm{cm}^{2}\left(8.4 \mu \mathrm{J} / \mathrm{cm}^{2}\right)$ to $7000 \mu \mathrm{J} / \mathrm{cm}^{2}\left(4200 \mu \mathrm{J} / \mathrm{cm}^{2}\right)$ and rescaled to set the saturated signal amplitude at $1500 \mathrm{ps}(2000 \mathrm{ps})$ to 1 . The plot of the rescaled signal amplitude as a function of excitation fluences (blue squares) and its fit to a Poisson statistics model (blue solid line), for (b) 4 ML CdSe and (d) CdSe/ZnS NPLs, respectively. See Supplementary Note for more details.

a

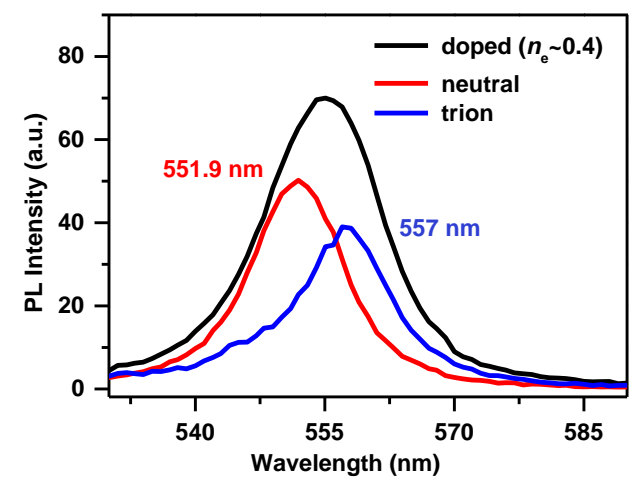

b

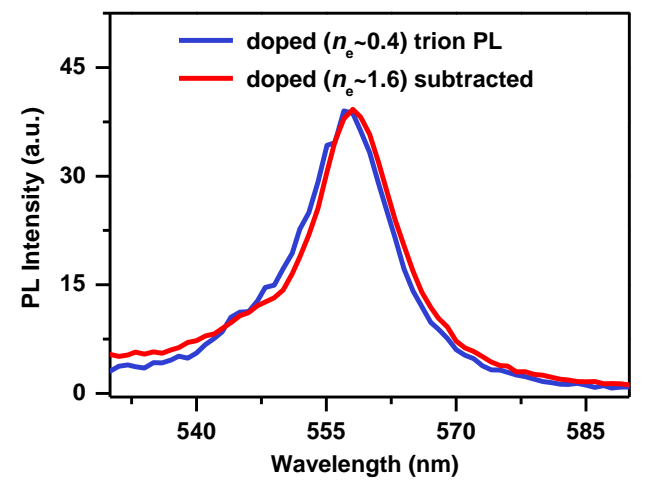

Figure S6. (a) Extracted PL spectra for pure negative trion (X', Peak $\sim 557 \mathrm{~nm})$ in 5

ML NPLs with an average electron doping number of $n_{\mathrm{e}}=0.4$. (b) Comparison of the PL spectra of charged species in 5 ML CdSe NPLs with $n_{\mathrm{e}}=0.4$ (blue line) and $n_{\mathrm{e}}=$ 1.6 (red line). 

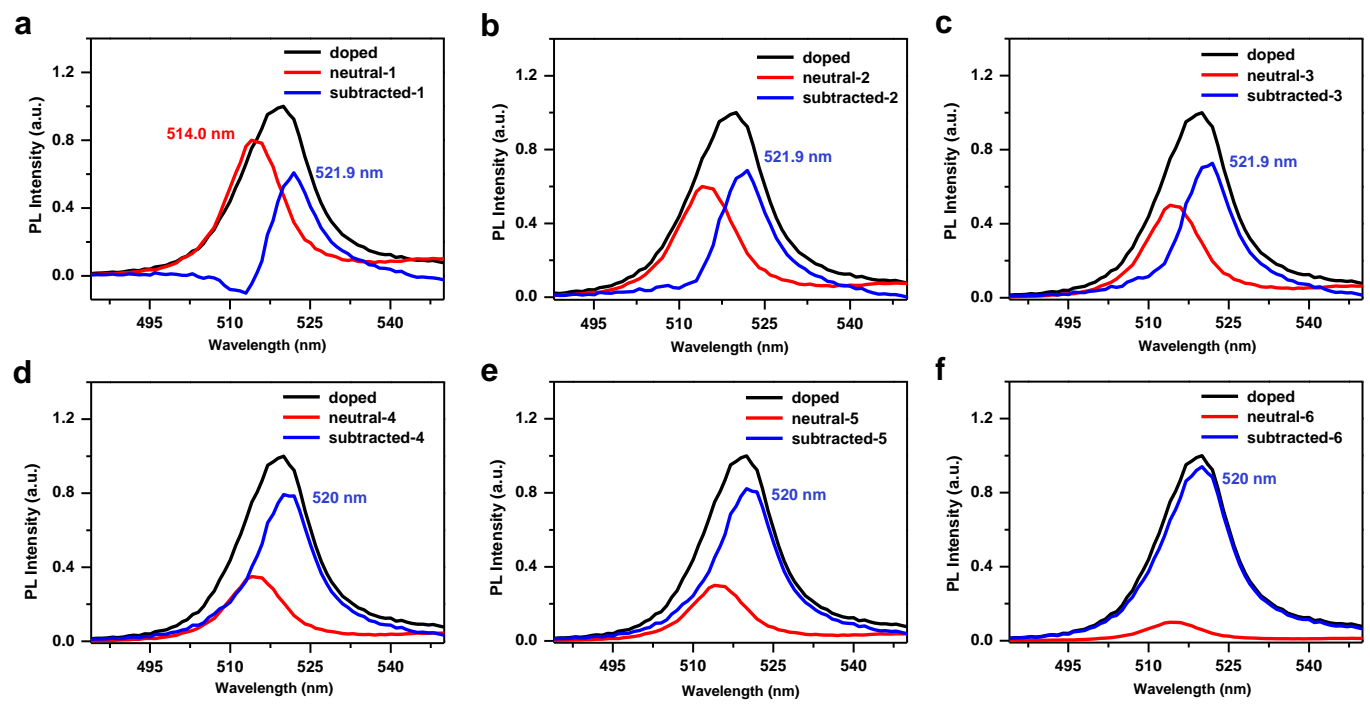

Figure S7. Determination of negative trion binding energy in $n$-doped 4 ML CdSe

NPLs. (a-f) With the decrease of the spectral amplitudes of neutral NPLs, the PL spectra of negative trion is slightly blue-shifted. Within this range, the error of the trion binding energy can be obtained.
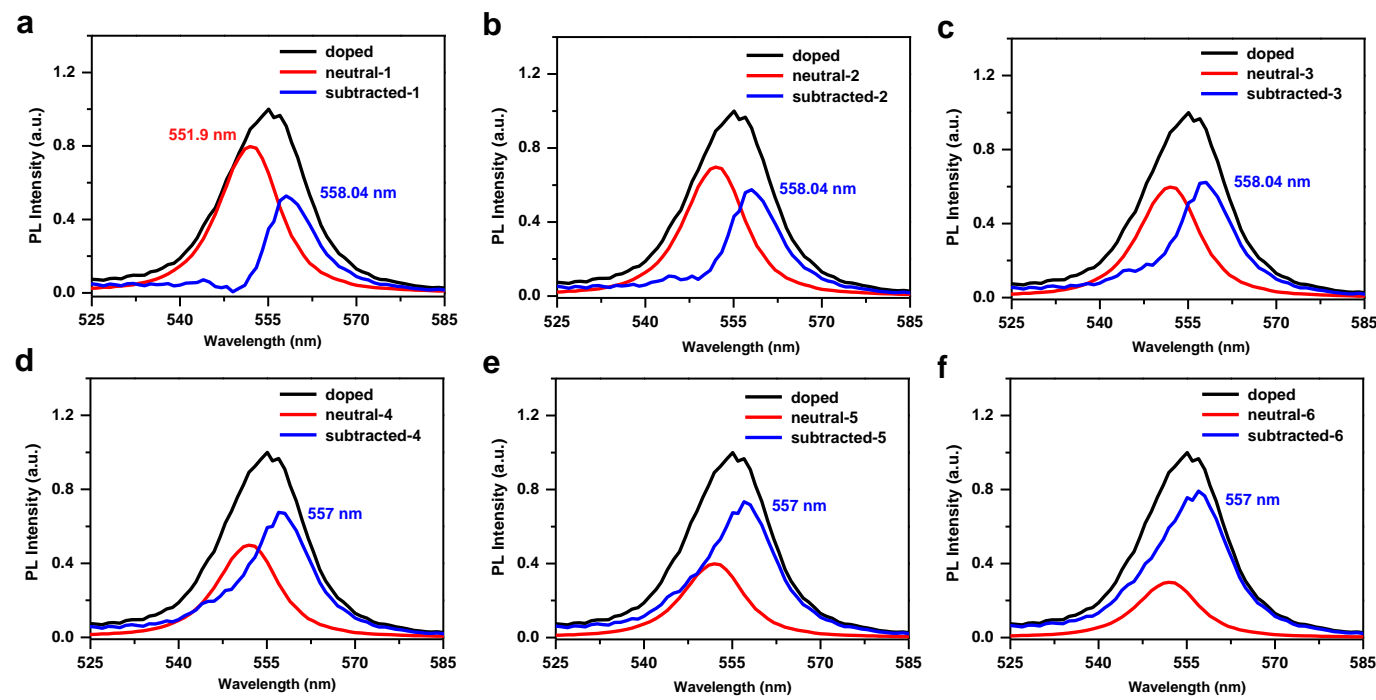

Figure S8. Determination of negative trion binding energy in $n$-doped $5 \mathrm{ML}$ CdSe NPLs. (a-f) With the decrease of the spectral amplitudes of neutral NPLs, the PL spectra of negative trion is slightly blue-shifted. Within this range, the error of the trion binding energy can be obtained. 

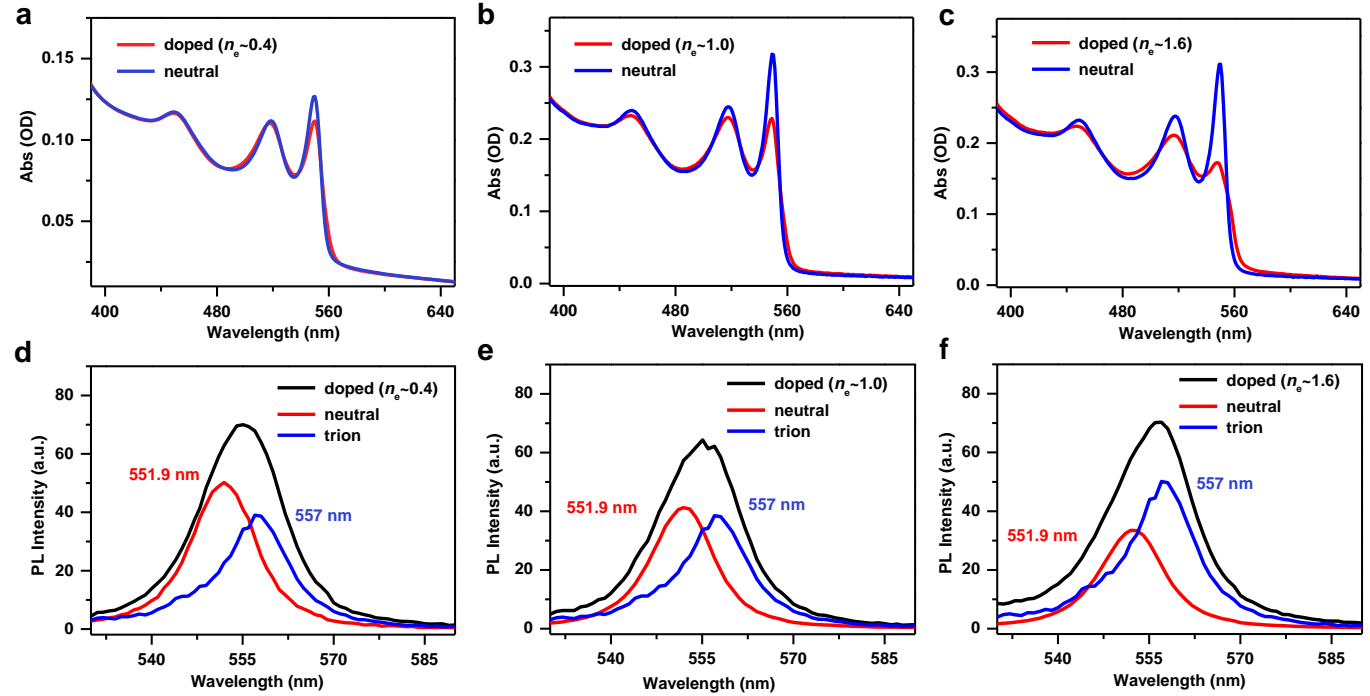

Figure S9. (a-c) Absorption spectra of $n$-doped and neutral NPLs with various doping levels for 5 ML CdSe NPLs. (d-f) Decomposition of the PL spectra into the exciton emission and trion emission in $n$-doped 5 ML CdSe NPLs with different doping levels.
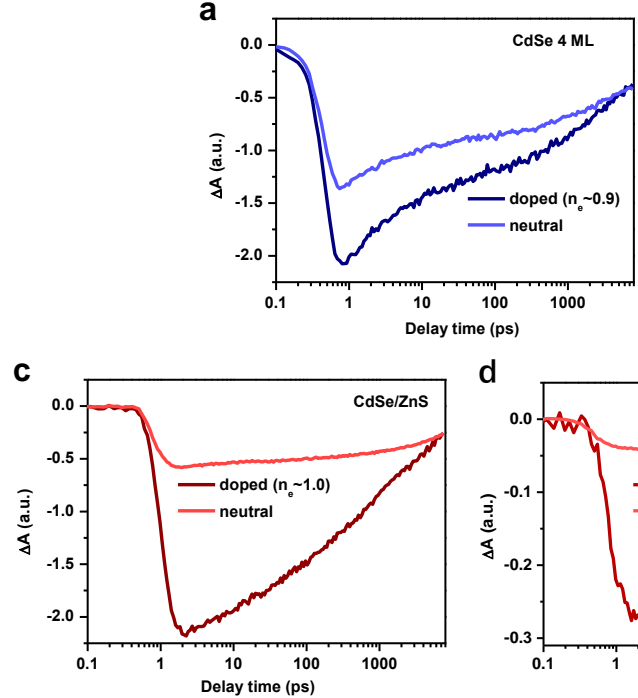

b
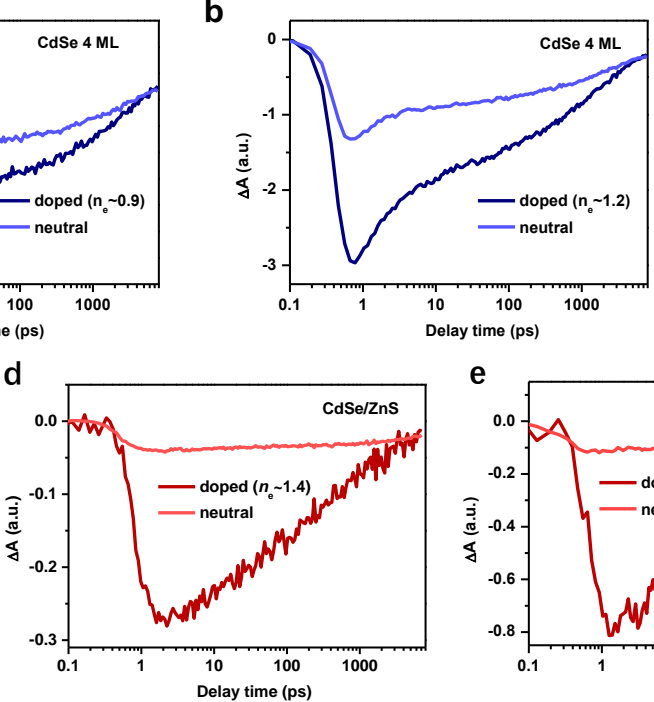

e

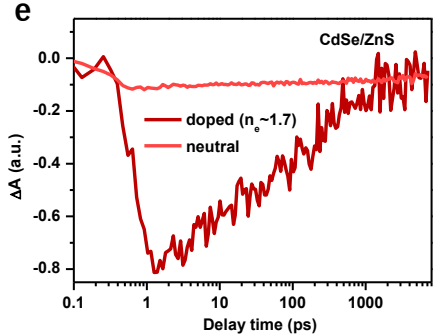

Figure S10. TA kinetics probed at the e-hh transition feature of various electron-doping levels for (a, b) 4 ML CdSe NPLs and (c, d, e) CdSe/ZnS NPLs respectively. The kinetics traces for $n$-doped (dark color) and neutral (light color) samples are normalized to the long-lived components. 

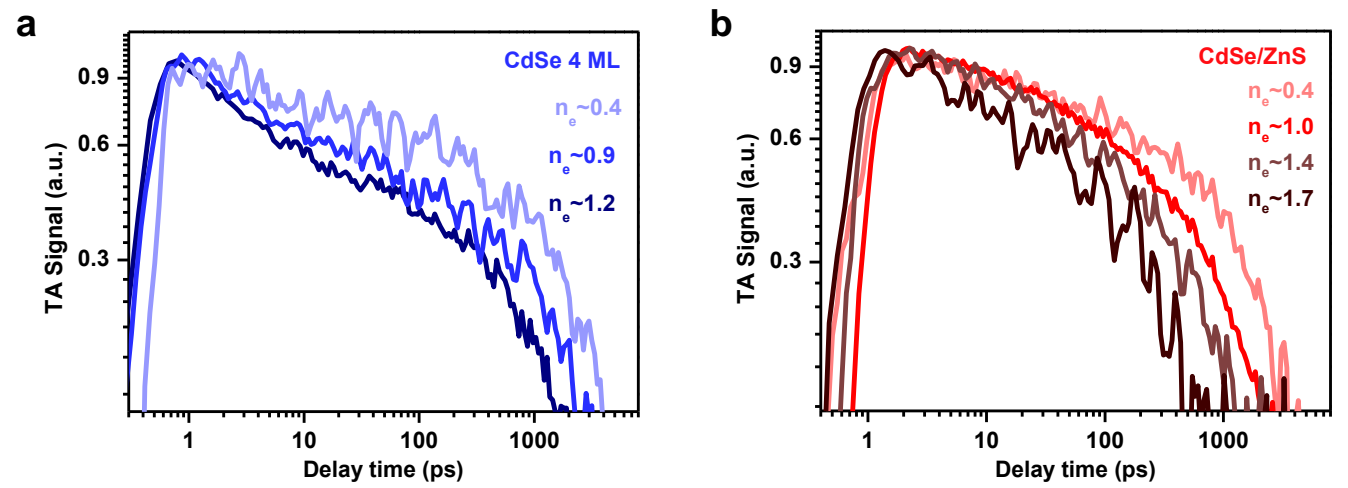

Figure S11. Charged exciton dynamics of various electron-doping levels for (a) $4 \mathrm{ML}$ CdSe NPL (blue lines) and CdSe/ZnS NPLs (red lines). The charged exciton recombination dynamics were obtained by performing a subtraction between the kinetics for $n$-doped and neutral NPLs in Figure S10.

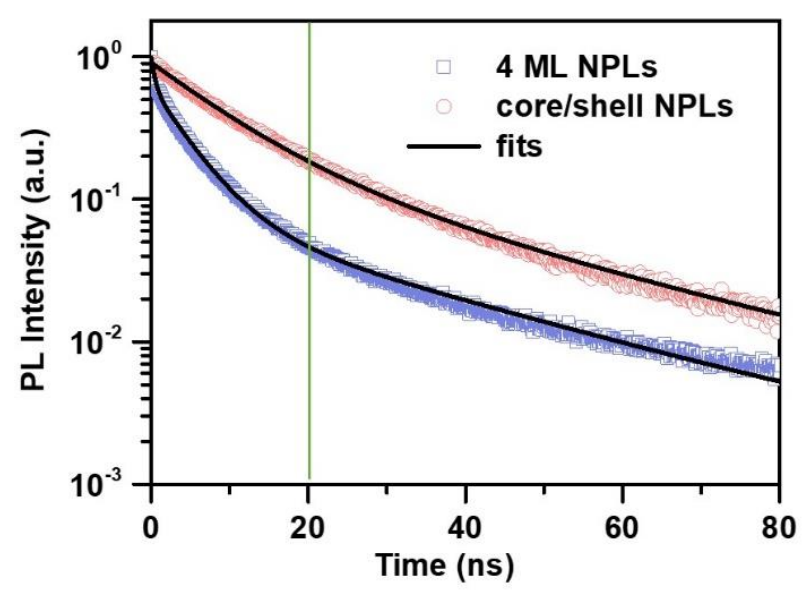

Figure S12. Time-resolved PL decay traces for 4 ML CdSe NPLs (blue squares) and $\mathrm{CdSe} / \mathrm{ZnS}$ core/shell NPLs (red circles) measured under $400 \mathrm{~nm}$ excitation and their fits (black solid lines). The decay for $4 \mathrm{ML}$ and core/shell NPLs is near three- and two-exponential with an average time constant of $5.0 \pm 0.1 \mathrm{~ns}$ and $14.0 \pm 0.1 \mathrm{~ns}$, respectively. 


\section{Sample preparations}

Preparation of cadmium myristate. Cadmium myristate was synthesized by following the recipe given in the literature with some modifications. ${ }^{1}$ Sodium myristate $(3.13 \mathrm{~g})$ was dissolved in $250 \mathrm{~mL}$ of methanol under strong stirring. Cadmium nitrate tetrahydrate $(1.23 \mathrm{~g})$ was dissolved in $40 \mathrm{~mL}$ of methanol and added dropwise to the sodium myristate solution, after which the reaction mixture was stirred by approximately two hours. The whitish product was washed with methanol five times for the removal of excess precursors. Subsequently, the final product was collected by filtration and dried under vacuum.

Synthesis of four monolayer (ML) CdSe Nanoplatelets. 4 ML CdSe nanoplatelets were synthesized according to the recipe given in the literature with slight modifications. ${ }^{1,2}$ For a typical synthesis, $170 \mathrm{mg}$ of cadmium myristate, $12 \mathrm{mg}$ of Se, and $30 \mathrm{~mL}$ of octadecene (ODE) were loaded into a $50 \mathrm{~mL}$ three-neck flask. The solution was degassed and stirred at $95{ }^{\circ} \mathrm{C}$ under vacuum for an hour to evaporate volatile solvents and dissolve the cadmium myristate completely. The heater was then set to $240{ }^{\circ} \mathrm{C}$.. When the temperature reached $100{ }^{\circ} \mathrm{C}$, the flask was switched from vacuum to nitrogen gas. As the temperature reached $200{ }^{\circ} \mathrm{C}$, the color of the solution became orange, and at this stage $40 \mathrm{mg}$ of cadmium acetate dihydrate $\left(\mathrm{Cd}(\mathrm{Ac})_{2} \cdot 2 \mathrm{H}_{2} \mathrm{O}\right)$ was introduced swiftly into the reaction. After the growth of CdSe NPLs at $240{ }^{\circ} \mathrm{C}$ for around 10 minutes, $1 \mathrm{~mL}$ of $\mathrm{OA}$ was injected when the temperature of the solution was decreased to $160{ }^{\circ} \mathrm{C}$ using air gun. Subsequently, $15 \mathrm{~mL}$ of hexane was injected when the solution was cooled to room temperature. The solution was centrifuged for 
$10 \mathrm{~min}$ at $3000 \mathrm{rpm}$, and the supernatant was transferred to another centrifuge tube. This solution was centrifuged at $7800 \mathrm{rpm}$ for $10 \mathrm{~min}$, and the precipitates were dissolved and stored in toluene as the final product.

Synthesis of 5 ML CdSe NPLs. For a typical synthesis, $170 \mathrm{mg}$ of cadmium myristate and $14 \mathrm{~mL}$ of ODE were loaded into a $50 \mathrm{~mL}$ three-neck flask. The solution was stirred and degassed at room temperature for half an hour and then at $95{ }^{\circ} \mathrm{C}$ for an hour under vacuum. The heater was then set to $250{ }^{\circ} \mathrm{C}$. When the temperature reached $100{ }^{\circ} \mathrm{C}$, the flask was switched from vacuum to nitrogen gas. When the temperature reached $250{ }^{\circ} \mathrm{C}$, a pre-prepared dispersion of $24 \mathrm{mg}$ Se dispersed in $2 \mathrm{~mL}$ of ODE was swiftly injected into the hot solution. When the color of solution turned into orange, $90 \mathrm{mg}$ of $\mathrm{Cd}(\mathrm{Ac})_{2} \cdot 2 \mathrm{H}_{2} \mathrm{O}$ was rapidly introduced. Subsequently, the solution was kept at $250{ }^{\circ} \mathrm{C}$ for $10 \mathrm{~min}$ and $1 \mathrm{~mL}$ OA was injected when the temperature decreased to $160{ }^{\circ} \mathrm{C}$ using air gun. Finally, $15 \mathrm{~mL}$ of hexane was added at room temperature. The mixture was then centrifuged at $3000 \mathrm{rpm}$ for 10 minutes, and the precipitate containing the NPLs was suspended in $30 \mathrm{~mL}$ hexane. To remove the by-products, a few drops of acetonitrile were added and centrifuged at $6000 \mathrm{rpm}$ for $30 \mathrm{~min}$. The precipitates were dissolved and stored in toluene.

Synthesis of CdSe/ZnS core/shell MPLs. CdSe/ZnS core/shell NPLs were synthesized following reported procedures with slight modifications. ${ }^{3} 0.2 \mathrm{mmol}$ zinc acetate, $7 \mathrm{~mL} 4 \mathrm{ML}$ CdSe NPLs (with optical density of 1.6 at $512 \mathrm{~nm}$ in $1 \mathrm{~mm}$ cuvette), $0.5 \mathrm{~mL} \mathrm{OA}$, and $5 \mathrm{~mL}$ ODE were added to a $50 \mathrm{~mL}$ three-neck flask. The solution was stirred under vacuum at room temperature for an hour to evaporate 
hexane. The mixture was then heated up to $90{ }^{\circ} \mathrm{C}$ and kept for 30 min to completely remove water and/or other remaining volatile solvents. After the degassing step, 0.5 $\mathrm{mL}$ oleylamine $(\mathrm{OL})$ was added into the solution under nitrogen flow. The reaction was then kept for $10 \mathrm{~min}$ and the temperature was set to $300{ }^{\circ} \mathrm{C}$. Octanethiol-ODE solution (87.5 $\mu \mathrm{L}$ octanethiol in $5 \mathrm{~mL}$ ODE) was prepared in glove box and injected into the reaction at $170{ }^{\circ} \mathrm{C}$ using a syringe pump with a speed of $8.85 \mathrm{~mL} / \mathrm{h}$. When the temperature reached $250{ }^{\circ} \mathrm{C}$, the injection speed of octanethiol-ODE solution was changed to $3.54 \mathrm{~mL} / \mathrm{h}$. The reaction was kept at $300{ }^{\circ} \mathrm{C}$ for $60 \mathrm{~min}$, which was then quenched by injection of $5 \mathrm{~mL}$ ODE and an ice-water bath. The product was washed with $20 \mathrm{~mL}$ ethanol and centrifuged at $5500 \mathrm{rpm}$ for $3 \mathrm{~min}$. The precipitated final product was dispersed in hexane.

\section{Photochemical doping of NPLs.}

Photochemical doping experiments followed reported procedures. ${ }^{4}$ Briefly, Li[Et 3 BH] (1 $\mathrm{M}$ solution in tetrahydrofuran) was diluted to $0.01 \mathrm{M}$ with toluene and was transferred together with NPLs in toluene into a glove box filled with $\mathrm{N}_{2}$ atmosphere (oxygen level $<0.2 \mathrm{ppm}$ ). The $\mathrm{Li}\left[\mathrm{Et}_{3} \mathrm{BH}\right]$ solution was added dropwise into the NPLs solution under vigorous stirring, which was then illuminated by a UV lamp to accelerate the reaction until it reached an equilibrated doped state. The degree of electron-doping was controlled by varying the amount of $\mathrm{Li}\left[\mathrm{Et}_{3} \mathrm{BH}\right]$ added and was monitored by UV-vis absorption spectra. The doped samples were sealed in custom-made airtight cuvettes (optical path $1 \mathrm{~mm}$ ) and transferred out of the glove box for all optical measurements. The neutralized samples were obtained by exposing 
the doped samples to the air for several minutes.

\section{TA and u-PL experimental setups}

TA measurements. The femtosecond pump-probe TA measurements were performed using a regenerative amplified Ti:sapphire laser system (Coherent; $800 \mathrm{~nm}, 70 \mathrm{fs}, 6$ $\mathrm{mJ} /$ pulse, and $1 \mathrm{kHz}$ repetition rate) as the laser source and a femto-TA 100 spectrometer (Time-Tech Spectra). Briefly, the $800 \mathrm{~nm}$ output pulse from the regenerative amplifier was split in two parts with a 50\% beam splitter. The transmitted part was used to pump a TOPAS Optical Parametric Amplifiers (OPA) which generated a wavelength-tunable laser from $250 \mathrm{~nm}$ to $2.5 \mu \mathrm{m}$ as pump beam. The reflected $800 \mathrm{~nm}$ beam was split again into two parts. One part with less than $10 \%$ was attenuated with a neutral density filter and focused into a $2 \mathrm{~mm}$ thick sapphire window to generate a white light continuum (WLC) used for probe beam. The probe was focused with an Al parabolic reflector onto the sample. After the sample, the probe beam was collimated and then focused into a fiber-coupled spectrometer with CMOS sensors and detected at a frequency of $1 \mathrm{kHz}$. The intensity of the pump pulse used in the experiment was controlled by a variable neutral-density filter wheel. The delay between the pump and probe pulses was controlled by a motorized delay stage. The pump pulses were chopped by a synchronized chopper at $500 \mathrm{~Hz}$ and the absorbance change was calculated with two adjacent probe pulses (pump-blocked and pump-unblocked). The samples were placed in $1 \mathrm{~mm}$ airtight cuvettes in $\mathrm{N}_{2}$-filled glove box and measure under ambient conditions. Samples were vigorously stirred in 
all measurements.

u-PL measurements. The femtosecond PL up-conversion (u-PL) experiments were performed on a Chimera spectrometer (Light conversion) using a Pharos laser (1030 $\mathrm{nm}, 100 \mathrm{kHz}, 230 \mathrm{fs}$ pulse-duration; Light conversion) as the excitation and gate sources. One part of the Pharos output was used to pump a TOPAS OPA to generate the wavelength-tunable excitation pulses, while the other was used as the gate pulse. The light emitted by the sample was collected by lens and focused into a BBO crystal together with the gate pulse to generate the up-converted signal via sum frequency generation (SFG). The signal photons were focused onto the entrance slit of a monochromator and detected by the spectrometer. The time-resolved decay curve was obtained by delaying the gate pulse with respect to the pump pulse using a mechanical decay stage. The samples were placed in $1 \mathrm{~mm}$ airtight cuvettes in $\mathrm{N}_{2}$-filled glove box and measure under ambient conditions. Samples were vigorously stirred in all measurements in order to minimize damaging of the samples.

\section{Supplementary Note 1: EMA calculation}

The effect of $\mathrm{ZnS}$ shell on conduction band minimum (CBM) and valence band maximum (VBM) was simulated with a single-band effective mass approximation model (EMA). As described above for Coulomb screening, the binding energy correction is mostly canceled out by the self-energy term, so we will only consider the confinement contribution in our simulation.

A model of a square plate in 3-region potentials and another one of 2-region 
potentials are employed for $\mathrm{CdSe} / \mathrm{ZnS}$ and CdSe nanoplatelets, respectively. The 3 regions from the center to the outside correspond successively to CdSe core, $\mathrm{ZnS}$ shell and oleate ligand layer, and their geometric parameters are all from the TEM patterns. The potentials were set as

$$
V=\left\{\begin{array}{cc}
0 & \text { CdSe core } \\
E_{C B, Z n S}\left(\text { or } E_{V B, Z n S}\right) & \text { ZnS shell } \\
E_{L U M O, O A}\left(\text { or } E_{\text {HOMO,OA }}\right) & \text { ligand shell }
\end{array}\right.
$$

where $E_{C B, Z n S}\left(\right.$ or $E_{V B, Z n S}$ ) is the energy offset between the CBMs (or VBMs) of bulk $\mathrm{ZnS}$ and $\mathrm{CdSe}$, and $E_{L U M O, O A}\left(\right.$ or $\left.E_{\text {Hомо,OA }}\right)$ is that between the LUMO (HOMO) of oleic acid and the CBM (VBM) of bulk CdSe. The CBM and VBM of bulk CdSe are both set to 0 in the simulation. The effective mass of the electron (hole) in the aforementioned three regions was defined as:

$$
m_{e(h)}^{*}=\left\{\begin{array}{cc}
m_{e(h), \text { CdSe }}^{*} & \text { CdSe core } \\
m_{e(h), Z n S}^{*} & \text { ZnS shell } \\
m_{0} & \text { ligand shell }
\end{array}\right.
$$

where $m_{0}$ is the free electron mass and $m_{e(h), C d S e}^{*}$ and $m_{e(h), Z n s}^{*}$ are the electron (hole) effective mass in bulk CdSe and $\mathrm{ZnS}$, respectively.

The parameters are summarized in Supplementary Table 1. The simulation was performed in COMSOL Multiphysics ${ }^{\circledR}$ Modeling Software using the semiconductor module. The calculations yielded a CBMs red-shifting $\sim 183 \mathrm{meV}$ and VBMs red-shifting $\sim 39 \mathrm{meV}$ respectively compared to free NPLs after shell coating, and a consequent overall red shift of $222 \mathrm{meV}$. 
Supplementary Table 1. Parameters for EMA calculation

\begin{tabular}{|l|c|c|c|c|}
\hline & $m_{e}^{*}\left(m_{0}\right)$ & $m_{h}^{*}\left(m_{0}\right)$ & $\begin{array}{c}\text { CBM/LUMO } \\
(\mathrm{eV} \text { vs vac })\end{array}$ & $\begin{array}{c}\text { VBM/HOMO } \\
(\mathrm{eV} \text { vs vac })\end{array}$ \\
\hline $\mathrm{CdSe}$ & 0.13 & 0.45 & -4.04 & -5.74 \\
\hline $\mathrm{ZnS}$ & 0.28 & 0.50 & -3 & -6.6 \\
\hline $\mathrm{OA}$ & 1 & 1 & -1 & -8.4 \\
\hline
\end{tabular}

\section{Supplementary Note 2: Calculation of band edge degeneracies for NPLs}

According to the Poisson statistics model described in Figure S5, the photo absorption events when using above band-gap excitation follows a Poisson distribution, ${ }^{5}$ and hence, the e-hh transition signal at a delay time of 1500 ps (after Auger recombination) can expressed as: $I \propto 1-P(0)=1-e^{-<N>}$, where $<N>$ is the average number of photons absorbed per NPLs. $\langle N\rangle$ is proportional to the pump laser fluence $j:\langle N\rangle=C j$. Therefore, the e-hh transition signal at $1500 \mathrm{ps}$ can be fitted with: $I \propto 1-e^{-C j}$, with $C$ as the only fitting parameter. Using the fitted $C$ value, the average exciton number $<N>$ at each pump fluence can be calculated (red dash lines in Figure S5b, 5d).

As described above, both amplitudes at early delay times (2-3 ps) and late delay times (1500-2000 ps) are saturated at high pump fluences, suggesting that all band-edge exciton states are fully occupied at early times and the multiexciton states have recombined to only a single-exciton state at late times. Therefore, the maximum bleach amplitude $(\sim 6.5)$ at $2-3$ ps corresponds to the exciton degeneracy of NPLs when normalized the TA spectra at late times to 1 . It should be pointed out that even at the lowest pump fluences $\left(28 \mu \mathrm{J} / \mathrm{cm}^{2}\right.$ and $8.4 \mu \mathrm{J} / \mathrm{cm}^{2}$ for $4 \mathrm{ML}$ and $\mathrm{CdSe} / \mathrm{ZnS}$ 
NPLs), with an estimated average exciton number per NPL of 0.22 and 0.04 respectively, the exciton bleach decay to $53 \%$ and $57.1 \%$ of its initial amplitude at 1500-2000 ps for 4 ML and CdSe/ZnS NPLs, separately. This decay accounts for both radiative and nonradiative decay of a single-exciton state, which should not be ignored to infer the exciton degeneracy for NPLs. Thus, the exciton degeneracy in the lateral area of NPLs is corrected to be 3.4 -fold $(6.5 \times 0.53)$ and 3.7 -fold $(6.5 \times 0.571)$ for $4 \mathrm{ML}$ and $\mathrm{CdSe} / \mathrm{ZnS}$ NPLs respectively. These exciton degeneracies are well consistent with the saturation number $(\sim 3.5)$ of band-edge excitons per NPL reported by a prior study, ${ }^{6}$ which can be explained by the similar lateral areas of the NPLs used therein and those studied here. We also note that the non-integer degeneracy is simply a result of ensemble average for NPLs with a heterogeneous distribution in their lateral areas.

\section{References for SI:}

1. Khan, A. H.; Pinchetti, V.; Tanghe, I.; Dang, Z.; Martin-Garcia, B.; Hens, Z.; Van Thourhout, D.; Geiregat, P.; Brovelli, S.; Moreels, I., Tunable and Efficient Red to Near-Infrared Photoluminescence by Synergistic Exploitation of Core and Surface Silver Doping of CdSe Nanoplatelets. Chem. Mater. 2019, 31 (4), 1450-1459.

2. Wu, K. F.; Li, Q. Y.; Jia, Y. Y.; McBride, J. R.; Xie, Z. X.; Lian, T. Q., Efficient and Ultrafast Formation of Long-Lived Charge-Transfer Exciton State in Atomically Thin Cadmium Selenide/Cadmium Telluride Type-II Heteronanosheets. ACS Nano 2015, 9 (1), 961-968.

3. Altintas, Y.; Quliyeva, U.; Gungor, K.; Erdem, O.; Kelestemur, Y.; Mutlugun, E.; 
Kovalenko, M. V.; Demir, H. V., Highly Stable, Near-Unity Efficiency Atomically Flat Semiconductor Nanocrystals of $\mathrm{CdSe} / \mathrm{ZnS}$ Hetero-Nanoplatelets Enabled by ZnS-Shell Hot-Injection Growth. Small 2019, 15 (8), 1804854.

4. Rinehart, J. D.; Schimpf, A. M.; Weaver, A. L.; Cohn, A. W.; Gamelin, D. R., Photochemical Electronic Doping of Colloidal CdSe Nanocrystals. J. Am. Chem. Soc. 2013, 135 (50), 18782-18785.

5. Klimov, V. I.; Mikhailovsky, A. A.; McBranch, D. W.; Leatherdale, C. A.; Bawendi, M. G., Quantization of multiparticle Auger rates in semiconductor quantum dots. Science 2000, 287 (5455), 1011-1013.

6. Li, Q.; Liu, Q.; Schaller, R. D.; Lian, T., Reducing the Optical Gain Threshold in Two-Dimensional CdSe Nanoplatelets by the Giant Oscillator Strength Transition Effect. J. Phys. Chem. Lett. 2019, 10 (7), 1624-1632. 